\title{
Synchronization of Chaotic Fractional-Order Lu-Lu Systems with Active Sliding Mode Control
}

\author{
Mohamad Reza Dastranj ${ }^{1}$, Aminreza Riahi ${ }^{2}$ and Mahboubeh Moghaddas ${ }^{3}$ \\ ${ }^{1}$ Young Researchers Club, Sabzevar Branch, Islamic Azad University, Sabzevar, Iran \\ ${ }^{2}$ Department of Electrical Engineering, Gonabad Branch, Islamic Azad University, \\ Gonabad, Iran \\ ${ }^{3}$ Young Researchers Club, Gonabad Branch, Islamic Azad University, Gonabad, Iran \\ ${ }^{1}$ mohamadrezadastranj@gmail.com,2a.riahi@ieee.org
}

\begin{abstract}
In this paper, synchronization of chaotic and Lu system has been done using the active sliding mode control strategy. Regarding the synchronization task as a control problem, fractional order mathematics is used to express the system and active sliding mode for synchronization. It has been shown that not only is the performance of the proposed method satisfying with an acceptable level of control signal, but also a rather simple stability analysis is performed. The latter is usually a complicated task for nonlinear chaotic systems
\end{abstract}

Keywords: fractional calculus; fractional order active sliding mode controller; synchronization; $\mathrm{Lu}-\mathrm{Lu}$

\section{Introduction}

Chaos synchronization is an important topic in the nonlinear science. Generally speaking, the synchronization phenomenon has the following feature: the trajectories of two systems (master and slave systems) are identical notwithstanding starting from different initial conditions. However, slight errors of initial conditions for chaos dynamical systems will lead to completely different trajectories. Therefore, the way to control two chaos systems for synchronization has received a great deal of interest in the past few decades.

Fractional calculus is a 300-year-old topic, but its applications to physics and engineering are just a recent focus of interest. Many systems are known to display fractional-order dynamics, such as viscoelastic systems [1], electrode-electrolyte polarization, and electromagnetic waves [2]. Most recently, many researchers have begun to investigate the chaotic dynamics of fractional-order systems. In Ref. [3], it has been shown that Chua's system of 2.7 order can produce a chaotic attractor. Ref. [4] pointed out that chaos can exist in the 2.4 order Rossler system and hyperchaos can also exist in the fractional-order Rossler system with order as low as 3.8. Ref. [5] studied the chaotic behaviors of 2.7-order unified chaotic system. Moreover, in the last decade it has been found that chaotic attractors indeed exist in fractional-order systems [6-10].

The question of stability is very important especially in control theory. In the field of fractional order control systems, there are many challenging and unsolved problems related to stability theory such as robust stability, bounded input - bounded output stability, internal stability, root-locus, robust controllability, robust observability, etc.

As a result of its advantages in practical application, synchronization in chaotic dynamic systems has attracted increasing attention from various research fields. A wide variety of methods have been proposed for synchronization of chaotic systems, including linear 
feedback control [11], sliding mode control [12], adaptive control [13] and so on. Most of the methods mentioned above are used to guarantee the asymptotic stability of chaotic systems. Among the fractional order controllers, the fractional order active sliding mode control (FOASMC) has been dealt with more than others. In this paper, we introduce a fractionalorder system chaoticchen. To control the synchronization of chaotic fractional-order system, an active sliding mode controller (ASMC) is proposed. This novel control rule makes the system states asymptotically stable. Simulation results show that the presented control method can easily eliminate chaos and stabilize the market. The rest of the paper is organized as follows:

\section{Fractional-Order Derivative and its Approximation}

\section{Definition}

Different integral operator, represented by $0^{D_{t}^{q}}$, is a combined differentiation-integration operator commonly used in fractional calculus and general calculus operator, including fractional-order and integer, which is defined as:

$$
0^{D_{t}^{q}}=\left\{\begin{array}{lr}
\frac{d^{q}}{d t} & q>0 \\
1 & q=0 \\
\int_{0}^{t}(d \tau)^{-q} & q<0
\end{array}\right.
$$

There are several definitions of fractional derivatives [14]. The best-known one is the Riemann-Liouvile definition, which is given by

$$
\frac{d^{q} f(t)}{d t^{q}}=\frac{1}{\Gamma} \frac{d^{n}}{d t^{n}} \int_{o}^{t} \frac{f(\tau)}{(t-\tau)^{q-n+1}} d \tau
$$

Where $\mathrm{n}$ is an integer such thatn $-1<q<n, \Gamma(0)$ is the Gamma function. The geometric and physical interpretation of the fractional derivatives was given as follows:

$$
\Gamma(z)=\int_{0}^{\infty} e^{-t} t^{z-1} d t
$$

The Laplace transform of the Riemann-Liouville fractional derivative is:

$$
L\left\{\frac{d^{q} f(t)}{d t^{q}}\right\}=s^{q} L\{f(t)\}-\sum_{k=o}^{n-1} s^{k}\left[\frac{d^{q-1-k} f(t)}{d t^{q-1-k}}\right]
$$

Where, L means Laplace transform, and $\mathrm{s}$ is a complex variable. Upon considering the initial conditions to zero, this formula is reduces to:

$L\left\{\frac{d^{q} f(t)}{d t^{q}}\right\}=s^{q} L\{f(t)\}$ 
The Caputo fractional derivative of order $\alpha$ of a continuous function $f: R^{+} \rightarrow R$ is defined as follows:

$$
\frac{d^{q} f(t)}{d t^{q}}=\left\{\begin{array}{cc}
\frac{1}{\Gamma(m-q)} \int_{0}^{t} \frac{f^{(m)}(\tau)}{(t-\tau)^{q-m+1}} & m-1<q<m \\
\frac{d^{m}}{d t^{m}} f(t) & q=m
\end{array}\right.
$$

Thus, the fractional integral operator of order $\alpha$ can be represented by the transfer function $\mathrm{H}(\mathrm{s})=\frac{1}{\mathrm{~s}^{\mathrm{q}}}$ in the frequency domain.

The standard definition of fractional-order calculus does not allow direct implementation of the fractional operators in time-domain simulations. An efficient method to circumvent this problem is to approximate fractional operators by using standard integer-order operators. In Ref.[15], an effective algorithm is developed to approximate fractional-order transfer functions, which has been adopted in [16] and has sufficient accuracy for time-domain implementations. In Table 1 of Ref [17], approximations for $1 / \mathrm{s}_{\mathrm{q}}$ with $\alpha$ from 0.1 to 0.9 in step 0.1 were given with errors of approximately $2 \mathrm{~dB}$. We will use the $1 / \mathrm{s}^{0.95}$ approximation formula [16] in the following simulation examples.

$\frac{1}{\mathrm{~s}^{95}} \approx \frac{1.2831 \mathrm{~s}^{2}+18.6004 \mathrm{~s}+2.0833}{1.2831 \mathrm{~s}^{3}+18.4738 \mathrm{~s}^{2}+2.6574 \mathrm{~s}+0.003}$

In the simulation of this paper, we use approximation method to solve the fractional-order differential equations.

\section{Designing the Fractional-order Active Sliding Mode Control and Analysis}

To design the active sliding mode controller, we have procedure a combination of the active controller and the sliding mode controller.

\section{Active Sliding Mode Controller Design}

Let us consider a chaotic fractional-order description of the system as it has been shown below:

$$
0^{D_{t}^{q}} X_{1}=A_{1} X_{1}+g_{1}\left(X_{1}\right) \quad 0<q<1
$$

Where $X_{1}(t)=\left(x_{1}, x_{2}, x_{3}\right)^{T}$ are real state vector, $A_{1} \in R^{3 \times 3}$ denotes the linear part of the system dynamics and $g_{1}: R^{3} \rightarrow R^{3}$ is nonlinear part of the system. Eq.(1) denotes the master system. Let $X_{0}=\left(x_{10}, x_{20}, x_{20}\right)^{T}$ be the any initial conditions in the chaos attractor of fractional-orders system (8). 
Now the controller $u(t) \in R^{3}$ is added to the slave system.

Thus:

$0^{D_{t}^{q}} X_{2}=A_{2} X_{2}+g_{2}\left(X_{2}\right)+u(t) \quad 0<\alpha<1$

That $\mathrm{X}_{2}, \mathrm{~A}_{2}$ and $\mathrm{g}_{2}$ implies the same roles as $\mathrm{X}_{1}, \mathrm{~A}_{1}$ and $\mathrm{g}_{1}$ for the master system. Synchronization of the systems means finding a control signal $u(t) \in R^{3}$ that makes state of the slave system to evolve as the states of the master system.

Now we define dynamic errors as below:

$0^{D_{t}^{q}} X_{2}-0^{D_{t}^{q}} X_{1}=A_{2} X_{2}+g_{2}\left(X_{2}\right)-A_{1} X_{1}-g_{1}\left(X_{1}\right)+u(t)$

Thus:

$0^{D_{t}^{q}} X_{2}-0^{D_{t}^{q}} X_{1}=A_{2} X_{2}+g_{2}\left(X_{2}\right)-A_{1} X_{1}-g_{1}\left(X_{1}\right)+u(t)$

That: $\quad e=X_{2}-X_{1}$ and $A_{1}=A_{2}=A$

Now we assume:

$G\left(X_{1}, X_{2}\right)=g_{2}\left(x_{2}\right)-g_{1}\left(x_{1}\right)+\left(A_{2}-A_{1}\right) x_{1}$

The aim is to design the controller $\mathrm{u}(\mathrm{t}) \in \mathrm{R}^{3}$ such that:

$\lim _{t \rightarrow \infty}\|e(t)\|=0$

Then we use the active control design procedure $[19,20] \mathrm{U}(\mathrm{t})$ change as following:

$u(t)=H(t)-G\left(X_{1}, X_{2}\right)$

Eq.(14) describes the newly defined control input $\mathrm{H}(\mathrm{t})$.

Where $H(t)$ is:

$H(t)=K w(t)$

Where $k \in R^{3}$ is a constant gain vector and $w(t) \in R$ is the control input that satisfies in:

$$
W(t)= \begin{cases}w^{+}(t) & s(e) \geq 0 \\ w^{-}(t) & s(e)<0\end{cases}
$$

Where $\mathrm{s}=\mathrm{s}(\mathrm{e})$ is a switching surface that describes the desired dynamics. The resultant error is then written by:

$$
0^{D_{t}^{q}} e=A e+K W(t)
$$

\section{Constructing a Sliding Surface}

Constructing a sliding surface which represents a desired system dynamics and the sliding surface has been described as follows: 
$s(e)=C e$

Where $C \in \mathrm{R}^{3}$ is a constant vector. An equivalent control is found when $\dot{S}(\mathrm{e})=0$ which is a necessary condition for the state trajectory to stay on the switching surface $S(e)=0$ onence, the controlled system satisfies the following conditions in the steady state:

$S(e)=0 \quad$ and $\quad \dot{S}(e)=0$

Based on equation (17) to (19), it could be deduced:

$\dot{S}(e)=\left(\mathrm{CO}^{D_{\mathrm{t}}^{\mathrm{q}}}(\mathrm{Ae}+\mathrm{kw}(\mathrm{t}))=0\right.$

Thus,

$0^{D_{t}^{q}} w(t)=-(c k)^{-1} C A\left(0^{D_{t}^{q}} e(t)\right)$

A solution of Eq.21. is:

$w_{e q}(t)=-(C K)^{-1} C A e(t)$

\section{Sliding Mode Control of Fractional Order System}

We consider the constant plus proportional rate reaching law, which will be considered in [18]. Accordingly, the reaching law is obtained as:

$$
0^{D_{t}^{q}} S=-\rho \operatorname{sgn}(s)-r s
$$

That $\operatorname{sgn}(0)$ represents the sign function. The $\rho, r$ are gains that the sliding conditions Eq.(19) is satisfied by. From Eqs. (17), (18) we have:

$$
0^{D_{t}^{q}} S=C 0^{D_{t}^{q}} e=C[A e+k w(t)]
$$

From Eqs. (23) and (24), we find control effort, which can be defined as:

$$
w(t)=-(C K)^{-1}[C(r I+A) e+\rho \operatorname{sgn}(s)] .
$$

\section{Stability}

First, we represent stability theorems from the fractional calculus.

Theorem 1 (Matignon [19]). The following system:

$$
0^{D_{t}^{q}}=A x, \quad x(0)=x_{0}
$$

Where $0<q<1, x \in \mathrm{R}^{\mathrm{n}}$ and $\mathrm{A} \in \mathrm{R}^{\mathrm{n} \times \mathrm{n}}$,is asymptotically stable if $|\arg (\mathrm{eig}) \mathrm{A}|>\mathrm{q} \pi / 2$.

According to Theorem 1, as long as all eigenvalues of $[\mathrm{A}-\mathrm{K}(\mathrm{CK}) \mathrm{C}(\mathrm{rI}+\mathrm{A})] \quad\left(\lambda_{\mathrm{i}}=\right.$ $1,2,3)$ satisfy the conditions $\left|\arg \left(\lambda_{\mathrm{i}}\right)\right|>\mathrm{q} \pi / 2$, the system is asymptotically stable. 


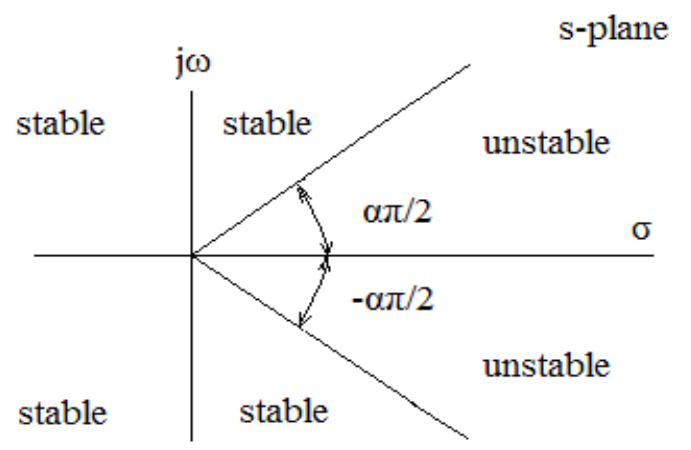

Figure 1. Stability Region of Linear Fractional-order System wih $\alpha$

\section{Numerical Simulations}

\section{Synchronization between Two Fractional-order Chen Systems}

The Lu system was introduced by Chen and Ueta in 1999 [20].

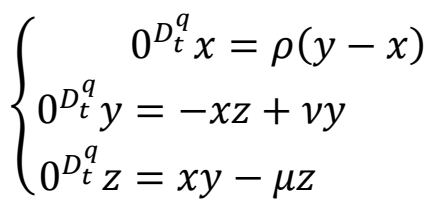

For this system matrix $\mathrm{A}$ is:

$A=\left[\begin{array}{ccc}-\rho & \rho & 0 \\ 0 & \nu & 0 \\ 0 & 0 & -\mu\end{array}\right]$

In this section, we consider using (ASMC) technique to obtain synchronization. This controller guarantees the synchronization of two fractional orders $\mathrm{Lu}$ systems with The following initial conditions:

$$
\begin{gathered}
\left(x_{10}, y_{10}, z_{10}\right)=(0,3,9) \\
\text { and } \\
\left(x_{20}, y_{20}, z_{20}\right)=(1,4,-10) .
\end{gathered}
$$

Consider two fractional order Lu systems as master and slave systems respectively:

$$
\begin{gathered}
\text { Master system }\left\{\begin{array}{c}
0^{D_{t}^{0.9}} x_{1}=35\left(y_{1}-x_{1}\right) \\
0^{D_{t}^{0.9}} y_{1}=-x_{1} z_{1}+28 y_{1} \\
0^{D_{t}^{0.9}} z_{1}=x_{1} y_{1}-3 z_{1}
\end{array}\right. \\
\text { Slave system }\left\{\begin{array}{c}
0^{D_{t}^{0.9}} x_{1}=35\left(y_{2}-x_{2}\right) \\
0^{D_{t}^{0.9}} y_{2}=-x_{2} z_{2}+28 y_{2} \\
0^{D_{t}^{0.9}} z_{2}=x_{2} y_{2}-3 z_{2}
\end{array}\right.
\end{gathered}
$$


Parameters of the controller are chosen as $\mathrm{k}=[-1.9,-4.8,-4]^{\mathrm{T}}, \mathrm{C}=[1,1,-1], \mathrm{r}=$ 66 and $\rho=0.43$. This selection of parameters results in eigenvalues $\left[\lambda_{1}, \lambda_{2}, \lambda_{3}\right]=$ $[-63,-65.4148,-6.5852]$ which:

located in a stable region $\left(\left|\arg \left(\lambda_{\mathrm{i}}\right)\right|\right)>\alpha \pi / 2$. Figure shows the effectiveness of the proposed controller to synchronize two fractional-order modeled systems. It should be noted that control $\mathrm{u}(\mathrm{t})$, has been activated at $\mathrm{t}=0$. The simulation results are shown in Figure 2.
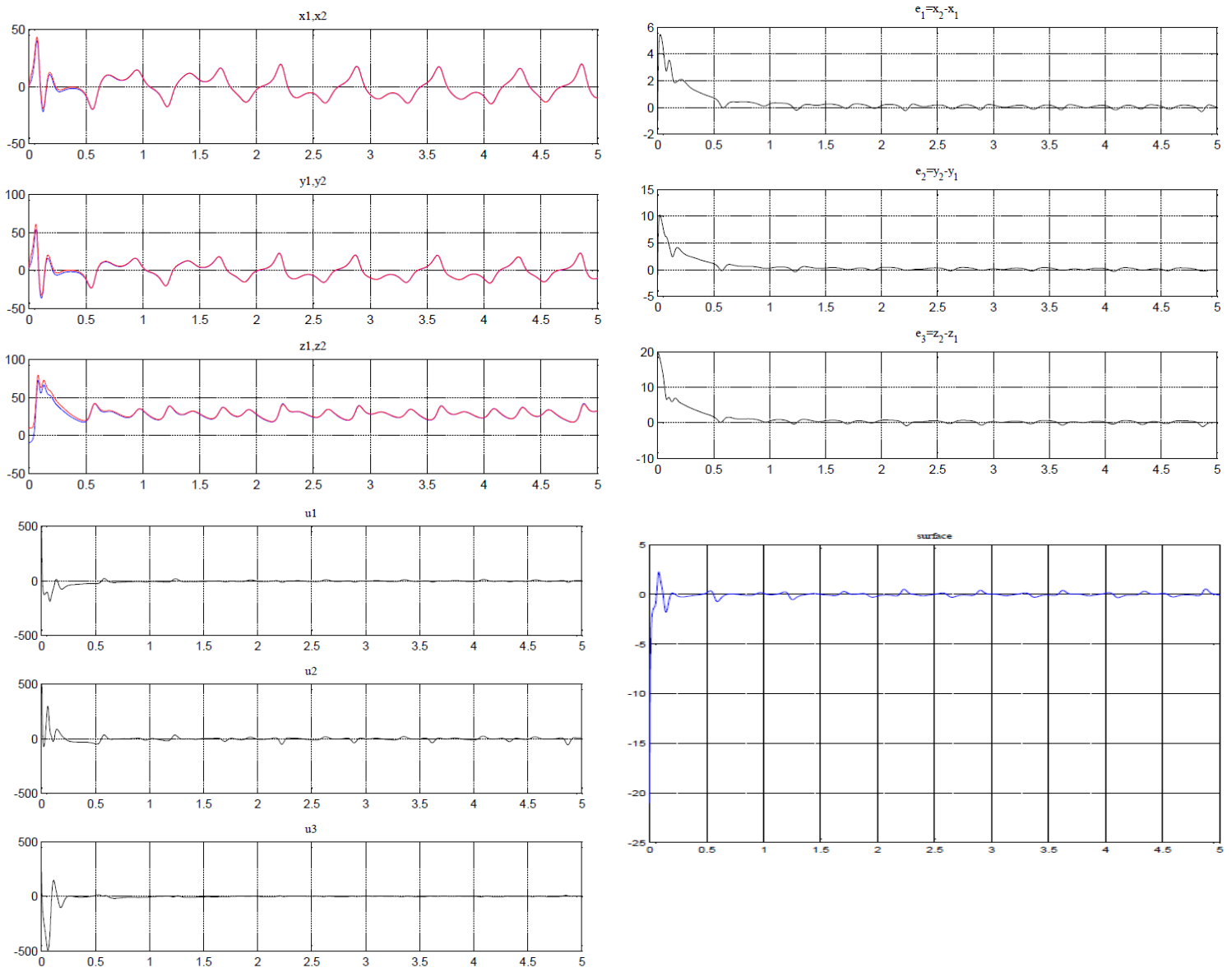

Figure 2. Results of Simulation

\section{Conclusion}

In this paper, we have studied numerical methods in fractional calculus. Then, we have represented the active sliding mode control to synchronization. The control parameters (r, k and c), the master and slave systems are synchronized. Numerical simulations show the efficiency of the proposed controller to synchronize chaotic fractional-order.

\section{References}

[1] P. L. Butzer, U. Westphal, “An introduction to fractional calculus, Singapore: World Scientific”, (2000).

[2] E. Ahmed and A. S. Elgazzar, "On fractional order differential equations model for nonlocal epidemics", Physica A, vol. 379, (2007), pp. 607-14.

[3] W. M. Ahmad and R. El-Khazali, "Fractional-order dynamical models of Love, Chaos Soliton Fract, vol. 33, no. 13 , (2007), pp. 67-75. 
[4] A. Le Me'haute', "Les Ge'ome' tries Fractales, Eidtions Herme` s", (1990);Paris, France.

[5] R. de Levie, "Fractals and rough electrodes, J. Electroanal. Chem.”, vol. 281, (1990), pp. 1-21.

[6] S. Westerlund, "Dead matter has memory! (capacitor model), Phys. Scrip." vol. 43, no. 2, (1991), pp. 174179.

[7] T. Kaplan, L. J. Gray and S. H. Liu, "Self-affine fractal model for a metal-electrolyte interface", Phys. Rev. B 35, vol. 10, (1987), pp. 5379-5381.

[8] E. Ott, C. Grebogi and J. A. Yorke, “Controlling chaos”, Physical Review Letters, vol. 64, no. 11, (1990), pp. 1196-1199.

[9] Y. G. Hong and J. K. Wang, "Finite time stabilization for a class of nonlinear systems", 18 8th International Conference on Control, Automation, Robotics and Vision, Kunming, (2004), pp. 1194-1199.

[10] S. Boccaletti, J. Kurths, G. Osipov, D.L. Valladares and C.S. Zhou, "The synchronization of chaotic systems", Physics Reports, (2002), pp. 366:1-101.

[11] F. Liu, Y. Ren, X. M. Shan and Z. L. Qiu, "A linear feedback synchronization theorem for a class of chaotic systems", Chaos, Solitons and Fractals, vol. 13, (2002), pp. 723-730.

[12] H. Zhang, X. K. Ma and W. Z. Liu, "Synchronization of chaotic systems with parametric uncertainty using active sliding mode control", Chaos, Solitons and Fractals, vol. 21, no.12, (2004), pp. 49-1257.

[13] H. Fotsin, S. Bowong and J. Daafouz, "Adaptive synchronization of two chaotic systems consisting of modified Van der Pol-Duffing and Chua oscillators", Chaos, Solitons and Fractals, vol. 26, (2005), pp. 215229.

[14] M. R. Dastranj, M. Rouhani and A. Hajipoor, "Design of Optimal Fractional Order PID Controller Using PSO Algorithm", International Journal of Computer Theory and Engineering 4, no. 3, (2012).

[15] A. Charef, H. H. Sun, Y. Y. Tsao and B. Onaral, "IEEE Trans. Auto. Contr., vol. 37, (1992), pp. 1465-1470.

[16] C. G. Li and G. R. Chen, "Chaos, Solitons \& Fractals, vol. 22, (2004), pp. 549-554.

[17] W. M. Ahmad and J. C. Sprott, "Chaos Solitons \& Fractals, vol. 16, (2003), pp. 339-351.

[18] H. Zhang, X. K. Ma and W. Z. Liu, "Synchronization of chaotic systems with parametric uncertainty using active sliding mode control", Chaos, Solitons Fractals, vol. 21, (2004), pp. 1249-1257.

[19] D. Matignon, "Stability results for fractional differential equations with applications to control processing", Computational Engineering in Systems and Application multi-conferenceIMACS, in: IEEE-SMC Proceedings, (1996); Lille, France.

[20] G. Chen and T. Ueta, "Yet another attractor, Int.", J. Bifurcation Chaos, vol. 9, (1999), pp.1465-1466.

\section{Authors}

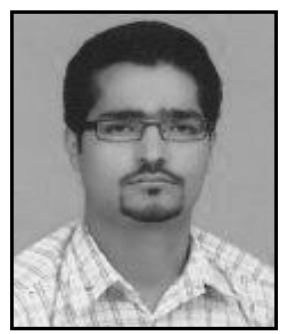

Mohamad Reza Dastranj graduated in B.S level from Islamic Azad University, Sabzevar branch, Iran in 2008. He graduated in Control Engineering, Islamic Azad University Gonabad branch, Iran in 2011 GONABAD. He is interested in the field of evolutionary algorithms and the fractional-order systems. The fact that he has published in international journal confirms his expertise in this research area.

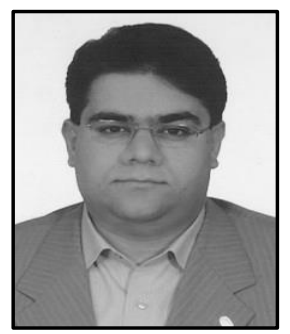

Aminreza Riahi received the B.S. degree in control \& automation engineering in 2011 at the Islamic Azad University, Gonabad Branch. Currently he is M.S. Student at the Islamic Azad University,Gonabad Branch and a member of Young Researchers and Elite Club IAU in Iran. His research interests focus on evolutionary algorithms, Fractional-order systems and modern control engineering. 